\title{
Amiodarone-Induced Thyroid Dysfunction and Ventricular Tachyarrhythmias During Long-Term Therapy in Japan
}

\author{
Tsuyoshi Shiga, MD; Michi Wakaumi, MD; Naoki Matsuda, MD; Morio Shoda, MD; \\ Nobuhisa Hagiwara, MD; Kanji Sato, MD*; Hiroshi Kasanuki, MD
}

\begin{abstract}
In 232 Japanese patients receiving long-term amiodarone therapy for life-threatening ventricular tachyarrhythmias, hyperthyroidism and hypothyroidism developed in 29 patients $(12.5 \%)$ and 25 patients $(10.8 \%)$, respectively. In patients with hyperthyroidism, the recurrence of sustained ventricular tachycardia was significantly higher with thyrotoxicosis than in the euthyroid period ( $31 \%$ vs $3 \%, \mathrm{p}<0.01)$. Holter monitoring showed that the average heart rate and ventricular premature complexes significantly increased with hyperthyroidism. On the other hand, there was no increase in the recurrence of ventricular tachyarrhythmia with hypothyroidism. There was no change in the dose or the plasma concentration of amiodarone or desethylamiodarone in the euthyroid period or when hyperthyroidism or hypothyroidism manifested. It is important to monitor for arrhythmia when hyperthyroidism develops during amiodarone therapy. (Jpn Circ J 2001; 65: 958-960)
\end{abstract}

Key Words: Amiodarone; Hyperthyroidism; Hypothyroidism; Ventricular tachycardia

A miodarone is an effective antiarrhythmic agent for life-threatening ventricular tachyarrhythmias, but it has a wide range of side effects, including thyroid dysfunction. Amiodarone has complex effects on thyroid physiology, and long-term therapy is associated with substantial changes in the results of standard thyroid function tests! Although most patients remain clinically euthyroid, $2-24 \%$ of patients develop either hyperthyroidism or hypothyroidism $!^{1-3}$ Thyroid hormones have been shown to alter cardiac excitability, affecting the myocardium directly and indirectly, which may lead to arrhythmias. There are 2 forms of amiodarone-induced hyperthyroidism: type 1, which develops in patients with latent Graves' disease or nodular goiters, and type 2, which develops in an apparently normal thyroid as a destructive thyroiditis. In Japan, most patients exhibit type 2 amiodarone-induced hyperthyroidism, which is different from the situation reported in Europe and the United States?

We evaluated whether amiodarone-induced thyroid dysfunction affected the antiarrhythmic effect of long-term therapy in Japanese patients with life-threatening ventricular tachyarrhythmias.

\section{Methods}

\section{Subjects and Study Design}

Between January 1987 and December 1998, 232 patients were referred for evaluation of ventricular tachycardia (VT) or fibrillation (VF) with organic heart disease and were subsequently treated with amiodarone. These patients

(Received May 16, 2001; revised manuscript received July 10, 2001; accepted July 30, 2001)

Department of Cardiology, The Heart Institute of Japan, and *Department of Medicine, Institute of Clinical Endocrinology, Tokyo Women's Medical University, Tokyo, Japan

Mailing address: Tsuyoshi Shiga, MD, Department of Cardiology, The Heart Institute of Japan, Tokyo Women's Medical University, 81 Kawada-cho, Shinjuku, Tokyo 162-8666, Japan. E-mail: mshiga@ hij.twmu.ac.jp were observed over a period of 2 years until December 2000, unless they died or there was discontinuation of the medication because of its side effects or inefficacy.

Monitoring of thyroid function (serum levels of thyroxine $\left(\mathrm{T}_{4}\right)$, free $\mathrm{T}_{4}$, triiodothyronine ( $\left.\mathrm{T}_{3}\right)$, free $\mathrm{T}_{3}$, and thyroid stimulating hormone (TSH)) and Holter monitoring were performed every 3 months during amiodarone therapy. Serum levels of $\mathrm{T}_{3}$ and $\mathrm{T}_{4}$ were determined with radioimmunoassay kits and those of free $\mathrm{T}_{3}$ and free $\mathrm{T} 4$ were determined with chemiluminescence assay kits. The serum level of TSH was determined with immunoradiometric assay kit5 Plasma concentrations of amiodarone and its active metabolite, desethylamiodarone, were measured by highperformance liquid chromatography 6 in some patients.

\section{Definition of Thyroid Dysfunction}

Amiodarone-induced hyperthyroidism can make it difficult to decide whether a patient is euthyroid or hyperthyroid, as the antiadrenergic effect of amiodarone can mask the clinical features of hyperthyroidism? Hyperthyroidism was defined as the presence of a serum TSH level of less than $0.1 \mu \mathrm{U} / \mathrm{ml}$ (normal range: $0.2-4.0 \mu \mathrm{U} / \mathrm{ml}$ ), in combination with high normal or high serum levels of $\mathrm{T}_{4}$, free $\mathrm{T}_{4}, \mathrm{~T}_{3}$ or free T32,5,7 Hypothyroidism was defined as a serum TSH level of $10 \mu \mathrm{U} / \mathrm{ml}$ or higher, in combination with low serum levels of $T_{4}$ or free $T_{4} 2,5,7$ Low serum levels of $T_{3}$ or free $T_{3}$ are an unreliable indicator of hypothyroidism as they may occur in euthyroid patients during amiodarone therapy!,2

\section{Recurrence of Ventricular Tachyarrhythmia}

Ventricular tachyarrhythmias were controlled with amiodarone in most patients prior to the diagnosis of thyroid dysfunction. We confirmed sustained VT or VF with or without symptoms by ECG recording or Holter monitoring, or by device recording in patients with an implantable cardioverter defibrillator.

\section{Statistics}

Baseline characteristics were compared between patients 
Table 1 Characteristics of Patients With and Without Amiodarone-Induced Thyroid Dysfunction

\begin{tabular}{lccc}
\hline \hline & $\begin{array}{c}\text { Without thyroid } \\
\text { dysfunction }\end{array}$ & Hyperthyroidism & Hypothyroidism \\
\hline No. of patients & 179 & 29 & 25 \\
Age (years) & $53 \pm 14$ & $51 \pm 14$ & $54 \pm 13$ \\
M/F & $147 / 32$ & $24 / 5$ & $18 / 7$ \\
Ischemic/nonischemic heart disease & $47 / 132$ & $9 / 20$ & $3 / 22$ \\
Ventricular tachycardia/fibrillation & $161 / 18$ & $25 / 4$ & $23 / 2$ \\
Left ventricular ejection fraction $(\%)$ & $36 \pm 17$ & $40 \pm 17$ & $39 \pm 14$ \\
Months since initiation of amiodarone & - & $32 \pm 12$ & $18 \pm 14^{*}$ \\
\hline
\end{tabular}

Values are expressed as mean $\pm S D . * p<0.001$ vs hyperthyroidism.

Table 2 Changes in the Recurrence of Sustained Ventricular Tachycardia (VT), Heart Rate and the Frequency of Premature Ventricular Complexes Obtained by Holter Monitoring, and in the Dose and Plasma Concentration of Amiodarone in the Euthyroid Period and at the Manifestation of Thyroid Dysfunction

\begin{tabular}{|c|c|c|c|c|}
\hline & \multicolumn{2}{|c|}{ Hyperthyroidism $(n=29)$} & \multicolumn{2}{|c|}{ Hypothyroidism $(n=25)$} \\
\hline & Euthyroid & At manifestation & Euthyroid & At manifestation \\
\hline Recurrence of sustained VT (cases) & 1 & $9 * *$ & 1 & 1 \\
\hline \multicolumn{5}{|l|}{ Holter monitoring } \\
\hline Mean heart rate (beats/min) & $65 \pm 8$ & $71 \pm 12 * *$ & $57 \pm 17$ & $61 \pm 15$ \\
\hline Premature ventricular complexes $(/ 24 h)$ & $806 \pm 1,736$ & $2,730 \pm 14,355^{*}$ & $1,573 \pm 2,562$ & $1,507 \pm 1,899$ \\
\hline Dose of amiodarone (mg/day) & $129 \pm 44$ & $132 \pm 45$ & $138 \pm 65$ & $142 \pm 51$ \\
\hline Plasma concentration & \multicolumn{2}{|c|}{$(n=20)$} & \multicolumn{2}{|c|}{$(n=19)$} \\
\hline Amiodarone $(\mu \mathrm{g} / \mathrm{ml})$ & $0.77 \pm 0.44$ & $0.68 \pm 0.28$ & $0.75 \pm 0.45$ & $0.71 \pm 0.30$ \\
\hline Desethylamiodarone $(\mu \mathrm{g} / \mathrm{ml})$ & $0.63 \pm 0.33$ & $0.54 \pm 0.23$ & $0.62 \pm 0.44$ & $0.59 \pm 0.32$ \\
\hline
\end{tabular}

Values are expressed as mean $\pm S D .{ }^{*} p<0.05,{ }^{* *} p<0.01$ vs euthyroid period.

without thyroid dysfunction, those with hyperthyroidism, and those with hypothyroidism by the Mann-Whitney test or one-way ANOVA. Recurrence of ventricular tachyarrhythmias was compared between within 6 months before (euthyroid period) and at the manifestation of hyperthyroidism or hypothyroidism by the Mann-Whitney test. Other parameters were compared between 3 and 6 months before (euthyroid period) and at manifestation of hyperthyroidism or hypothyroidism by the paired t-test. A p value less than 0.05 was considered significant.

\section{Results}

Twenty-nine patients $(12.5 \%)$ were diagnosed as having hyperthyroidism and $25(10.8 \%)$ as having hypothyroidism. One patient developed hyperthyroidism 12 months and then hypothyroidism 30 months after beginning amiodarone. There was no significant difference in patient background (ie, age, sex, underlying heart disease, type of VT, and left ventricular ejection fraction) among patients without thyroid dysfunction, those with hyperthyroidism, and those with hypothyroidism. Hypothyroidism developed earlier than hyperthyroidism after amiodarone was initiated (Table 1), as has been described in Europe and the United States!

In patients with hyperthyroidism, the recurrence of sustained VT was significantly higher with thyrotoxicosis than in the euthyroid period (Table 2). Neither VF nor sudden cardiac death was observed in these patients. Holter monitoring showed that the average heart rate and ventricular premature complexes significantly increased with hyperthyroidism, but no correlation was found between serum levels of free $\mathrm{T}_{3}$ and free $\mathrm{T}_{4}$ at the manifestation of hyperthyroidism and changes in frequency of ventricular premature complexes when hyperthyroidism developed versus the euthyroid period. No significant difference was found in these thyroid hormone values between patients with and without sustained VT. The dose of amiodarone when hyperthyroidism occurred was the same as that in the euthyroid period. There was no change in the plasma concentration of amiodarone or desethylamiodarone in the euthyroid period or with hyperthyroidism. No significant difference was found in these concentrations between patients with and without sustained VT.

On the other hand, in patients with hypothyroidism, there was no increase in the recurrence of VT. Holter monitoring showed that the average heart rate and ventricular premature complexes did not change. The dose of amiodarone when hypothyroidism occurred was the same as that in the euthyroid period and there was no change in the plasma concentration of amiodarone or desethylamiodarone (Table 2).

Of the patients with hyperthyroidism, 6 were treated with methimazole and 2 were treated with prednisolone. The other patients improved spontaneously in a few months without treatment, although 3 of these patients had to have amiodarone discontinued because of frequent VT $(n=2)$ or pulmonary complication $(n=1)$. The remaining patients continued amiodarone treatment. Twelve of the patients with hypothyroidism were supplemented with L-thyroxine and none of them discontinued amiodarone.

\section{Discussion}

Amiodarone-induced hyperthyroidism is reported to have a lower incidence in patients living in iodine-sufficient areas: 3 , However, the present results show that amiodarone causes hyperthyroidism with a comparatively high incidence in Japan, which has relatively greater iodine sufficiency than the United States. Although sufficient intake of iodine may account for the rare incidence of type 1 hyperthyroidism, 5 the pathogenesis of amiodarone-induced hyper- 
thyroidism in Japan is not clear.

Patients with hyperthyroidism often develop atrial arrhythmias because the atria are more sensitive than the ventricles to the effect of thyroid hormones?,10 This preferential atrial arrhythmogenic effect of thyroid hormones seems to be caused by various factors such as atrial $\beta$ adrenergic receptor density ${ }^{11}$ or differences between atrial and ventricular autonomic innervation and differences in sensitivity4 However, hyperthyroidism associated with organic heart disease or marked heart failure can cause malignant arrhythmias, including ventricular tachycardia and fibrillation. No definite relationship was found in this study between serum levels of thyroid hormones when hyperthyroidism manifested and the occurrence of ventricular arrhythmia, although it has been reported that a weak correlation exists between Lown grades of ventricular arrhythmia and $\mathrm{T}_{4}$ levels in patients with hyperthyroidism. ${ }^{10}$ However, it is difficult to demonstrate a simple relationship between serum levels of thyroid hormones and ventricular arrhythmia because amiodarone inhibits the peripheral conversion of $\mathrm{T}_{4}$ to $\mathrm{T}_{3}$, which may lead to elevated $\mathrm{T}_{4}$ levels during long-term amiodarone therapy? It has been reported that hyperthyroidism is heralded by the reappearance of arrhythmia if it was initially controlled by amiodarone!-4,12

The mechanisms of the cardiovascular effect of thyroid hormones seem to be as follows: (1) directly affecting the myocardium by stimulating specific nuclear receptors, which accelerate the production of specific mRNAs, and by changing the transcription of numerous muscle genes encoding both myofibrillar and calcium-regulatory proteins ${ }^{3}$; (2) affecting the myocardium through the sympathetic nervous system, which alters the response to sympathetic stimulation by modulating adrenergic receptor function and density? The sympathetic nervous system plays a role in the occurrence of life-threatening ventricular arrhythmia and so hyperthyroidism can precipitate ventricular arrhythmias by stimulating sympathetic nervous system activity. The significant increase in ventricular premature complexes and heart rate in hyperthyroidism seen in our patients could be caused not only by the direct chronotropic effect of thyroid hormones on the myocardium, but also by independent stimulation of the sympathetic nervous system.

Most Japanese patients exhibit type 2 amiodaroneinduced hyperthyroidism as a destructive thyroiditis, ${ }^{5}$ possibly in response to interleukin-63,13 Rapid release of thyroid hormone because of the destructive thyrotoxicosis may be one of the factors that accelerate arrhythmogenesis. The precipitation of arrhythmia, however, does not seem to be caused by a reduced effect of amiodarone itself, because neither the dose nor plasma concentration of amiodarone showed any significant change during the euthyroid period or at manifestation of hyperthyroidism. Furthermore, because no significant difference was observed in plasma concentrations at the manifestation of hyperthyroidism between patients with and without sustained ventricular tachycardia, it seems unlikely that the occurrence of ventricular arrhythmia is caused by changes in the pharmacokinetics of amiodarone. On the other hand, amiodaroneinduced hypothyroidism did not increase ventricular arrhythmias, and it has been suggested that hypothyroidism might protect against arrhythmias because they are rarely encountered in patients with hypothyroidism. Hypothyroidism prolongs the cardiac action potential and the QT interval $1{ }^{14}$ and is considered to be one of the mechanisms of the antiarrhythmic effect of amiodarone 4,15

\section{Conclusion}

Hyperthyroidism, but not hypothyroidism, which develops during long-term amiodarone treatment of life-threatening ventricular tachyarrhythmias, has been found to precipitate arrhythmia in Japan. It is important to monitor thyroid function periodically during amiodarone treatment and carefully monitor for arrhythmia when hyperthyroidism develops. Hyperthyroidism can be predicted when an increase in heart rate or recurrence of arrhythmia is observed.

\section{References}

1. Newman CM, Price A, Davies DW, Ray TA, Weetman AP: Amiodarone and the thyroid: A practical guide to the management of thyroid dysfunction induced by amiodarone therapy. Heart 1998; 79: $121-127$

2. Harjai KJ, Licata AA: Effects of amiodarone on thyroid function. Ann Intern Med 1997; 126: 63-73

3. Klein I, Ojamaa K: Thyroid hormone and the cardiovascular system. N Engl J Med 2001; 344: 501-509

4. Polikar R, Burger AG, Scherrer U, Nicod P: The thyroid and the heart. Circulation 1993; 87: 1435-1441

5. Sato K, Miyakawa M, Eto M, Inaba T, Matsuda N, Shiga T, et al: Clinical characteristics of amiodarone-induced thyrotoxicosis and hypothyroidism in Japan. Endocrine J 1999; 46: $443-451$

6. Flanagan RJ, Storey GCA, Holt DW: Rapid high-performance liquid chromatographic method for measurement of amiodarone in blood plasma or serum at the concentrations attained during therapy. $J$ Chromatogr 1980; 187: 391-398

7. Toft AD, Boon NA: Thyroid disease and the heart. Heart 2000; 84: $455-460$

8. Martino E, Safran M, Aghini-Lombardi F, Rajatanavin R, Lenziardi M, Fay M, et al: Environmental iodine intake and thyroid dysfunction during chronic amiodarone therapy. Ann Intern Med 1984; 101: $28-34$

9. Freedberg AS, Papp JG, Vaughan Williams EM: The effect of altered thyroid state on atrial intracellular potentials. J Physiol 1970; 207: 357-369

10. Olshausen KV, Bischoff S, Kahaly G, Mohr-Kahaly S, Erbel R, Beyer J, et al: Cardiac arrhythmias and heart rate in hyperthyroidism. Am J Cardiol 1989; 63: 930-933

11. Golf S, Løvstad R, Hansson V: $\beta$-adrenoceptor density and relative number of $\beta$-adrenoceptor subtypes in biopsies from human right atrial, left ventricular, and right ventricular myocard. Cardiovasc Res 1985; 19: 636-641

12. Nademanee K, Singh BN, Callahan B, Hendrickson JA, Hershman JM: Amiodarone, thyroid hormone indexes, and altered thyroid function: Long-term serial effects in patients with cardiac arrhythmias. Am J Cardiol 1986; 58: 981-986

13. Bartalena L, Brogioni S, Grasso L, Bogazzi F, Burelli A, Martino E: Treatment of amiodarone-induced thyrotoxicosis, a difficult challenge: Results of a prospective study. J Clin Endocrinol Metab 1996; 81: $2930-2933$

14. Ojamaa K, Sabet A, Kenessey A, Shenoy R, Klein I: Regulation of rat cardiac Kv1, 5 gene expression by thyroid hormone is rapid and chamber specific. Endocrinology 1999; 140: 3170-3176

15. Nademanee K, Singh BN, Hendrickson JA, Reed AW, Melmed S, Hershman J: Pharmacokinetic significance of serum reverse T3 levels during amiodarone treatment: A potential method for monitoring chronic drug therapy. Circulation 1982; 66: 202-211 